\title{
A DOENÇA EM CADA SÉCULO: A INFLUÊNCIA DO COMPORTAMENTO SOCIAL NAS PRINCIPAIS PANDEMIAS DOS ÚLTIMOS 200 ANOS
}

DISEASE IN EVERY CENTURY: the influence of social behavior in the main pandemics of the last 200 years

ENFERMEDAD EN CADA SIGLO: la influencia del comportamiento social en las principales pandemias de los últimos 200 años

\section{Fernanda Oliveira Brito dos Reis ${ }^{* 1}$, Maria Sortênia Alves Guimarães ${ }^{2}$, Adolpho Dias} Chiacchio ${ }^{3}$, Neilton Araujo de Oliveira ${ }^{4}$

${ }^{1}$ Discente do Curso de Medicina, Universidade Federal do Tocantins (UFT). Palmas, TO, Brasil. ${ }^{2}$ Docente do Curso de Medicina; Mestre em Ciências da Saúde e Doutora em Ciências da Nutrição, Universidade Federal do Tocantins (UFT). Palmas, TO, Brasil.

${ }^{3}$ Docente do Curso de Medicina, Universidade Regional de Gurupi (UNIRG). Gurupi, TO, Brasil.

${ }^{4}$ Docente do Curso de Medicina e do Mestrado Profissional em Ciências da Saúde; Mestre em Saúde Coletiva e Doutor em Ciências, Universidade Federal do Tocantins (UFT). Palmas, TO, Brasil.

*Correspondência:fernanda.brito@mail.uft.edu.br

\section{Artigo recebido em 13/06/2020 aprovado em 03/11/2020 publicado em 12/03/2021.}

\section{RESUMO}

O ser humano e a doença sempre caminharam lado a lado durante toda a história da humanidade. Na maior parte desta trama, o homem conseguiu prevalecer sobre as infecções ocasionadas por agentes patogênicos e não patogênicos, com perdas e ganhos na evolução da vida e dos povos, entretanto, algumas epidemias/pandemias desestabilizam a homeostase humana e, assim, promovem desequilíbrios. Este artigo, a partir de uma revisão narrativa da literatura, traz um panorama de algumas das maiores epidemias mundiais dos três últimos séculos, com as principais características e acontecimentos históricos de cada época, ao tempo em que apresenta e correlaciona a influência do comportamento humano na propagação e desenvolvimento delas, especificamente a Cólera, Gripe Espanhola e COVID-19, e aponta algumas das muitas lições que foram e que ainda podem ser aprendidas.

Palavras-chave: cólera; coronavírus; influenza pandêmica 1918-1919.

\section{ABSTRACT}

Human beings and disease have always gone hand in hand throughout human history. In most of history, man was able to prevail over infections caused by pathogenic and non-pathogenic agents, with losses and results of the evolution of life and peoples, however, some epidemics/pandemics destabilize human homeostasis and, therefore, promote imbalances. This article, based on a narrative review of the literature, provides an overview of some of the greatest global epidemics of the last three centuries, with the main resources and historical histories of each era, the time when it presents and correlates the activity of human behavior in their propagation and development, specified in Cholera, Spanish Flu and COVID-19, and points out some of the many editions that can still be learned.

Keywords: cholera; coronavírus; influenza pandemic, 1918-1919. 


\section{RESUMEN}

Los seres humanos y las enfermedades siempre han ido de la mano a lo largo de la historia humana. En la mayor parte de esta trama, el hombre pudo prevalecer sobre las infecciones causadas por agentes patógenos y no patógenos, con pérdidas y ganancias en la evolución de la vida y los pueblos, sin embargo, algunas epidemias / pandemias desestabilizan la homeostasis humana y, por lo tanto, promueven desequilibrios. Este artículo, basado en una revisión narrativa de la literatura, proporciona una visión general de algunas de las mayores epidemias mundiales de los últimos tres siglos, con las principales características y eventos históricos de cada época, al tiempo que presenta y correlaciona la influencia del comportamiento humano en su propagación y desarrollo, específicamente el cólera, la gripe española y COVID-19, y señala algunas de las muchas lecciones que se han aprendido y se pueden aprender.

Descriptores: cólera; coronavírus; influenza pandémica, 1918-1919.

\section{INTRODUÇÃO}

As doenças, parte intrínseca na história da humanidade, constituem terreno fértil para problematizar, evocar, representar questões socioculturais (Pascual, 2017). Já no ano 428 a. C, a Peste de Atenas (Rezende et al., 2018) e, ao longo do tempo, outras epidemias se mostraram potenciais agentes destrutivos de civilizações, não somente pelo número espantoso de mortes, como também pela desestruturação econômica, política e social (Gonzatto et al., 2020). Diversos historiadores defendem que o contexto no qual as doenças surgem tem papel fundamental para entender o seu curso e seu impacto na sociedade (Jones, 2020).

Para além da dimensão tradicional da doença (a tríade epidemiológica clássico hospedeiro, patógeno e meio ambiente), toda enfermidade é praticamente um iceberg da história, visto pelas modernas percepções científicas e biomédicas (Schrecker, 2012). Omran, na década de 1970, explicava que as mudanças ocorrem em função do binômio -ser humano e moléstia-, fato conhecido como teoria das transições epidemiológicas mudanças complexas nos padrões do processo saúdedoença, além das interações entre esses padrões, com seus determinantes sociais, econômicos, socioculturais, políticas e demográficos, assim como suas consequências (Omran, 2005).

Nos últimos dois séculos perdurou a segunda transição epidemiológica, caracterizada pelo aumento da densidade populacional e da expectativa de vida, diminuição da incidência de doenças agudas e elevação da prevalência de doenças crônicas, um cenário resultante de vários fatores, como o desenvolvimento da medicina, melhorias nutricionais provenientes da expansão agrícola e medidas de saúde pública que contribuíram para o declínio da mortalidade Já no século XXI, está em curso a terceira transição epidemiológica, com persistência de doenças crônicas e reemergência de doenças infecciosas, associadas a uma resistência, cada vez mais ampla, aos antibióticos e, pela presença de comorbidades, maior gravidade e disseminação, através dos cada vez mais modernos sistemas de transporte de pessoas e de produtos, no processo de globalização (Harper e Armelagos, 2010).

Alguns componentes influenciam substancialmente o surgimento, curso e desfecho das doenças infecciosas, dos quais destacam-se os fatores comportamentais e/ou sociais. Estudos realizados por Funk et al.(2010) apontam que o comportamento humano, constituído por atitudes, crenças, opiniões e conscientização sobre a ameaça infecciosa, pode ter 
um impacto direito no modelo Suscetível-InfectadoRecuperado (SIR). Embora a modificação do comportamento social, seja crucial para o desfecho de uma pandemia, isto não se apresenta de maneira homogênea (Ferguson, 2007). Fatores como a rede social, diferenças socioeconômicas e tradições são responsáveis pelo andamento diferente de uma doença pelo mundo (Bauch e Galvani, 2013).

O componente tempo é muito relevante, pois as doenças de disseminação acelerada são naturalmente mais assustadoras, com modelação mais pontual do comportamento, talvez pelo medo ser tão contagioso quanto à própria moléstia (Lodge et al., 2020; Epstein, 2009) ao contrário do que ocorre com doenças insidiosas, quando se tem um comportamento elástico e protetor, à medida que aumenta o número de casos (Funk et al., 2010). Logo, as percepções de risco também são um componentechave das teorias de mudança comportamental (Barrios, 2020).

A influência do comportamento da sociedade, no contexto de grandes pandemias é pouco discutida e, até onde foi possível constatar, poucos estudos tratam de evidências diretas sobre a adaptação comportamental em diferentes epidemias, sociedades e contextos geográficos (Lodge et al.,2020). Desse modo, é preciso esquadrinhar a história e as características dos países e cidades, do trabalho humano, das mudanças socioambientais e comportamentais, das guerras e das viagens, pois todas estas variáveis estão associadas entre si (Herzlich, 2004).

O presente estudo realiza uma análise críticoreflexiva das influências do comportamento humano/social no surgimento, curso e desfecho de três grandes pandemias dos últimos três séculos.

\section{MATERIAIS E MÉTODOS}

Trata-se de um artigo de revisão narrativa de literatura, realizada de fevereiro a abril de 2020, numa perspectiva ampla do tema para provocar reflexão e debate. Este tipo de revisão mapeia o estado da arte em um tópico de pesquisa, permitindo estabelecer relações entre produções anteriores, identificar temáticas recorrentes e apontar novas perspectivas (Vosgerau, 2014). Este método é adequado para discutir aspectos teórico-conceituais, bem como o contexto de implantação, de modo articulado (Green et al.,2006).

A revisão consistiu em três fases: (i) reconhecimento do contexto histórico do surgimento, curso e desfecho de três grandes pandemias nos últimos três séculos, (ii) identificação e síntese dos conhecimentos relativos à influência do comportamento humano/social no transcurso de doenças epidêmicas, objeto central desse estudo; (iii) análise crítica do comportamento humano social no contexto histórico das pandemias.

As buscas foram feitas nas Bases de Dados da Biblioteca Virtual em Saúde (BVS), Lilacs (Literatura Latino-Americana em Ciências da Saúde), Medline (Literatura Internacional em Ciências da Saúde), SciELO (Scientific Electronic Library Online), além de livros disponibilizados digitalmente e outros sites, especialmente, do Ministério da Saúde (MS) e Organização Mundial da Saúde (OMS).

Os trabalhos foram escolhidos em três etapas: análise de títulos, dos resumos e dos textos completos, não se levando em consideração o tempo de publicação dos mesmos, face à necessidade da coleta de dados históricos sobre os assuntos.

Na consulta, utilizou-se o descritor Pandemia conjugado com Cólera, Influenza Pandêmica, 
Coronavírus e Comportamento Social, e seus similares em inglês e espanhol (Tabela 1).

No total, 85 trabalhos foram selecionados, dos quais 40 foram analisados pormenorizadamente e organizados conforme os eventos, como mostra a Tabela 2.

Tabela 1. Síntese dos descritores utilizados na pesquisa e suas respectivas traduções para o inglês e espanhol.

\begin{tabular}{|c|c|c|}
\hline Português & Inglês & Espanhol \\
\hline $\begin{array}{l}\text { Pandemias+ } \\
\text { Cólera }\end{array}$ & $\begin{array}{l}\text { Pandemics + } \\
\text { Cholera }\end{array}$ & $\begin{array}{l}\text { Pandemias + } \\
\text { Cólera }\end{array}$ \\
\hline $\begin{array}{l}\text { Pandemias + } \\
\text { Influenza } \\
\text { Pandêmica }\end{array}$ & $\begin{array}{l}\text { Pandemics }+ \\
\text { Influenza Pandemic }\end{array}$ & $\begin{array}{l}\text { Pandemias + } \\
\text { Influenza } \\
\text { Pandémica }\end{array}$ \\
\hline $\begin{array}{l}\text { Pandemias + } \\
\text { Coronavírus }\end{array}$ & $\begin{array}{l}\text { Pandemics + } \\
\text { Coronavirus }\end{array}$ & $\begin{array}{l}\text { Pandemias }+ \\
\text { Coronavirus }\end{array}$ \\
\hline $\begin{array}{l}\text { Pandemias+ } \\
\text { Comportament } \\
\text { o Social }\end{array}$ & $\begin{array}{l}\text { Pandemics }+ \text { Social } \\
\text { Behavior }\end{array}$ & $\begin{array}{l}\text { Pandemias }+ \\
\text { Conducta Social }\end{array}$ \\
\hline
\end{tabular}

Tabela 2. Quantidade dos artigos, analisados em profundidade, e sua identificação com os eventos estudados.

\begin{tabular}{ccccc}
\hline Eventos & Cólera & $\begin{array}{c}\text { Gripe } \\
\text { Espanhola }\end{array}$ & COVID-19 & $\begin{array}{c}\text { História \& } \\
\text { Comportamento } \\
\text { humano }\end{array}$ \\
\hline $\begin{array}{c}\text { Quantidade de } \\
\text { artigos }\end{array}$ & 4 & 8 & 6 & 22 \\
\hline
\end{tabular}

Alguns destes estudos dizem respeito exclusivamente a uma das pandemias, enquanto os outros 22 (vinte e dois) versam sobre História, Epidemiologia \& Comportamento Humano em Pandemias, abrangendo os aspectos sociais, culturais, psicológicos, políticos e econômicos e, 1 (um) destes, reporta tanto sobre História \& Comportamento
Social, quanto a respeito da Cólera e da Gripe Espanhola.

\section{RESULTADOS E DISCUSSÃO}

\section{A (5ª) PANDEMIA DE CÓLERA - Século XIX (1881 a 1896)}

O século XIX foi um período de inúmeras mudanças e um divisor de águas na produção de ideias, por isso considerado por Michael Foucault como "o século da história" (Mattos, 2009).

No campo da ciência, esteve presente a emergente consolidação da Teoria dos Germes pela qual várias doenças obtiveram a identificação dos seus agentes causais (White, 2006). A sociedade também se transformou, com a adoção do sistema capitalista, substituindo a subjetividade da marcação das horas pelos sinos das igrejas, por um controlado e impessoal tempo marcado pelo relógio das fábricas (Mattos, 2009). A distância entre os países ficou cada vez menor, devido à intensificação dos contatos entre o Velho e o Novo Mundo e instalação de ferrovias na Europa, América do Norte e Índia, além do uso do transporte de navios a vapor (White, 2006; Santos, 1994), avanços usufruídos pelas classes nobres da época, em virtude da desigualdade social que imperava. A população carente vivia em aglomerações e precárias condições de higiene, sem saneamento e sem água potável, formando ambiente propício para a instalação de doenças, em especial as infectocontagiosas, como a Cólera (White, 2006; Wokaunn et al., 2012).

Causada pelo Vibrio cholerae, a doença iniciou em 1817 um ciclo de seis pandemias durante todo o século XIX (Pollitzer, 1959). Aqui nos interessa apenas a quinta pandemia, em virtude de peculiaridades históricas. 
O termo Cólera existe desde o século V a.C, através de registros de Hipócrates e acredita-se que sua etimologia foi derivada do grego, significando "fluxo da bile" (Pollitzer, 1959; Macpherson, 1884; Howard-Jones, 1979). Todavia, apenas a partir de 1849, com John Snow, pensou-se que problemas gastrointestinais, com milhares de mortes, poderiam ser consequência de uma fonte de transmissão contaminada, como a água consumida pelos habitantes. Anterior a isso, predominava a teoria miasmática, limitando as autoridades, médicos e cidadãos de alterarem seu comportamento e de promoverem prevenção de doenças transmissíveis. Acreditava-se, então, que as mortes com sintomas coléricos -vômitos incoercíveis, dores abdominais e diarreias exorbitantes- eram causadas pelo ar contaminado proveniente de pântanos, de cadáveres e lixões (Santos, 1994).

Essas crenças também impediram que a Cólera fosse diagnosticada mais cedo, visto que o microrganismo "Cholerigenic vibrios" - assim nomeado em 1854 pelo médico Filippo Pacini, que o descobriu -, fora ignorado pela comunidade científica (Pacini, 1854). Somente no quinto surto mundial de cólera, em 1881 no sul da Ásia, Robert Koch, apoiador da teoria dos germes, identificou o microrganismo em formato de vírgula nos tecidos de pacientes doentes e defendeu uma reformulação no isolamento de pessoas contaminadas (White, 2006).

Embora seus achados estivessem corretos, não foram amplamente aceitos, pois muitos duvidavam que uma bactéria poderia ser causa suficiente para desenvolver um processo patológico (White, 2006), em algumas cidades, médicos eram vistos como exterminadores das comunidades carentes, em razão de suas medidas serem salubres (Gonzalez, 1999), a crença popular dominante era de que cenários de moléstia eram castigo divino, podendo ser tratado por uma medicina doméstica, baseada na religiosidade (Santos, 1994; Wokaunn et al., 2012). O conhecimento de dados dessa quinta pandemia é limitado, mas o empirismo dos tratamentos oferecidos na época, como enemas, óleos, cloreto de mercúrio (colomel) e sangria, possivelmente contribuíram para um maior número de mortes (Vicentini et al.,2011). Acredita-se que um dos fatores preponderantes para a disseminação da Cólera - além das peregrinações muçulmanas a Meca, as emigrações por causa da fome, da movimentação nos portos e da pobreza -, foi a ineficiente fiscalização dos Estados (Pascual,2017). A Inglaterra, um dos poucos países da Europa a não sofrer com a pandemia, estava à frente do seu tempo com infraestrutura minimamente eficiente $\mathrm{e}$ reconheceu a existência de um microrganismo, naquela época nomeado "germe", aumentando as inspeções e relatórios, enquanto que as medidas mais adotadas pelo resto do mundo foram as Neocontagionistas de Peter Baldwin, com desinfecção de bens e pessoas, isolamento de doentes e instalação de hospitais específicos para a Cólera (White, 2006).

As grandes epidemias da história, como é o caso da Cólera, conforme William McNeill, desencadearam importantes rupturas históricas e provocaram choques violentos em vários âmbitos da sociedade, por vezes, decorrentes da própria rigidez das autoridades políticas, da ciência, da medicina e da população. Ademais, a demora em unir forças científico/médicas, para uma organização sanitária e uma formulação de protocolos específicos no contingenciamento do vibrião, repercutiu na morte de milhares de cidadãos, principalmente os de baixa 
renda, imigrantes e escravos em países colonizados, como o Brasil (Santos, 1994).

A responsabilidade por altos índices de contaminação não deve recair unicamente sobre as autoridades da época, a população alimentava fervorosamente preconceitos e estigmas sobre determinados grupos sociais, que a impedia de perceber que a Cólera não era exclusiva de solteiros, alcoólatras, pobres e pessoas com distúrbios mentais (como se acreditava na época), pelo contrário, atingia a todos e necessitava de mudanças de comportamento que acompanhassem os feitos do século (Rodriguez, 1889).

Num breve apanhado histórico vê-se a influência comportamental, cultural/religiosa numa pandemia e, quando as autoridades governamentais e de saúde carecem de credibilidade perante à população, torna-se mais trabalhoso empreender esforços para conscientizar e mobilizar a sociedade sobre medidas de contingenciamento da doença. Transmitir informações oportunas à sociedade, e com clareza, objetividade e simplicidade, pode ser o ponto central no enfrentamento e superação de uma pandemia.

\section{A PANDEMIA DE GRIPE ESPANHOLA - Século XX (1918-1919)}

$\mathrm{O}$ século XX iniciou com a consolidação de importantes mudanças ocorridas ainda no século XIX. A revolução industrial, a partir de 1850, trouxe inovações que transformaram diversos setores da sociedade. A ciência, até então, vivia seu ápice devido ao sucesso das descobertas na área da microbiologia (Silveira, 2005). O telefone e o telégrafo reinventaram o modo e a velocidade de comunicação das classes nobres e abastadas, assim como as ferrovias encurtaram trajetos. $\mathrm{O}$ aço e eletricidade foram descobertos, a mineração e a extração de petróleo impulsionado pela demanda das novas indústrias, com máquinas a vapor se instalando por toda a Europa. Pela necessidade de mão de obra nas fábricas, as cidades tornaram-se mais populosas e novos métodos de produção ditariam o ritmo dos trabalhadores (Chalton e Macarfle, 2017).

Com isso, na primeira década do século XX, conhecida como Belle Époque, pensava-se que o progresso havia chegado ao seu ápice. Esses anos dourados podem ser considerados presentes, porém, somente entre 1870 a 1914 , pois essas e outras mudanças exigiram demandas por matéria-prima, esgotada na expansão econômica e sociopolítica das grandes potências da Europa, como Alemanha e, também, no caso da Inglaterra, pelo medo da perda de mercado consumidor e do domínio territorial, derivando uma corrida imperialista, que logo culminou na grande guerra de 1914 (Chalton e Macarfle, 2017).

A Primeira Guerra Mundial, cujo fim se deu em novembro de 1918, ocasionou a morte de cerca de 15 milhões de pessoas (Chalton e Macarfle, 2017). É claro que a pandemia surgida no decorrer da guerra, e que matou duas vezes mais do que o próprio conflito e um terço do que a peste dizimou ao longo de 600 anos, contribuiu para que o tratado de paz fosse assinado (Sequeira, 2001).

A Gripe Espanhola, que até então não possuía este nome, não tinha seu agente etiológico conhecido. O primeiro caso foi registrado em março de 1918 nos Estados Unidos (White, 2006) e o termo "espanhola" não denota o epicentro da doença, supõe que se deva à eficiente notificação do número de doentes na pandemia, pela Espanha, que inclusive estava neutra na guerra (Rezende, 2018). 
Por aproximadamente um ano a moléstia assolou o mundo, num contágio em forma de três ondas. Os infectados tinham sintomas inespecíficos e variados, "o que confundia com Dengue, Tifo, Cólera e a Peste" (Tognotti, 2003; Crosby, 1999; Kolata, 2000). Cerca de um terço da população mundial da época foi atingida e entre 20 a 50 milhões de pessoas foram dizimadas, principalmente a população jovem, construindo um padrão dos óbitos em "W" (menores de 5 anos, 20-40 anos e maiores de 65 anos), muitos, associados a infecções secundárias, como pneumonia (Short et al., 2018; Taubenberger e Morens, 2006). As bases teóricas da bacteriologia estremeceram-se, pois não se dispunha de aparatos tecnológicos para determinar a presença de um então vírus (o H1N1, sabe-se hoje). Contudo, a teoria de Pfeiffer de que a devastadora doença era causada pelo Haemophilus influenza foi aceita na época, mesmo com muitas discordâncias (White, 2006). Só a partir de 1931, exatamente em 1933, com a criação do microscópio eletrônico é identificado o agente etiológico da gripe (Monto e Webster, 2013).

Seja qual for o caso, o H1N1 aproveitou o espaço criado pelo homem para fazer a transição do surto local para uma pandemia global (Flecknoe et al., 2018). Fatores sociais como aglomerações e falta de higiene nas trincheiras da guerra, transporte de soldados em navios, déficit nutricional com escassez de alimentos, inexistência dos antibióticos (descobertos em 1928, por Alexander Fleming), falta de médicos nas cidades, formaram um ambiente propício à sua disseminação (Nickol e Kindrachuk, 2019). Essa disseminação não decorreu unicamente do conflito bélico, apesar de sua forte contribuição. Países como Alasca e Samoa, distantes da contenda, tiveram casos da enfermidade (White, 2006).
$\mathrm{O}$ posicionamento da população, frente à gripe, teve grande influência. O ímpeto irresponsável pela guerra levou autoridades, militares e cidadãos a promoverem desfiles para arrecadar fundos pra guerra, contrariando medidas de contenção e isolamento, expedidas pelos conselhos de saúde (Barry, 2004; Holmberg, 2017), mas, por sua vez, a aceitação progressiva da existência de um microrganismo, possibilitou uma democratização dos "culpados" da Gripe Espanhola (Gurgel, 2013). A flexibilização da dominação religiosa perante a ciência favoreceu a desmistificação da culpabilidade dos feiticeiros e grupos religiosos (Bertucci, 2009; Bertucci, 2004) e, então, uma lenta e gradativa mudança de mentalidade possibilitou a compreensão de que todos eram responsáveis pela transmissão da influenza (Gurgel, 2013).

Só na década de 1990 se conseguiu sequenciar o genoma do vírus da gripe espanhola, a partir de material colhido de cadáveres no Alasca que contraíram o vírus (White, 2006). Hoje é sabido que as gripes, antes de 1918 relatadas como "febre catarral", "catarro epidêmico" e "destempero" (Delacy, 1993), eram causadas pelo mesmo vírus (H1N1).

Essa pandemia não foi o primeiro e nem a último registro de surtos por esse agente etiológico, desde o século XVIII ocorreram seis gripes documentadas. Todavia, é tida como a "mãe de todas as pandemias" face ao número de mortos num período de tempo tão curto e pelo achado de material genético da mesma cepa de vírus, em outros surtos de gripe que ocorreram posteriormente (Taubenberger e Morens, 2006).

Com o fim da pandemia, dúvidas e questionamentos foram levantados acerca do futuro e sobre como o mundo deve se comportar para afastar 
nova catástrofe em nível planetário. Com lições aprendidas pela sociedade e com novas conquistas, nos 100 anos de pós Gripe Espanhola, com novas formas de organização do Estado em diferentes países, esforços consideráveis da medicina e da ciência e enormes avanços tecnológicos, uma nova pandemia em 2020, outra vez ameaça a humanidade. Cabe, então, uma reflexão a respeito do comportamento da população mundial frente aos fatos, dados e aprendizados deixados na história, e fica a pergunta: quando a responsabilidade de prevenir pandemias, entendida como sendo de governos, laboratórios de pesquisa, vacinas e medicamentos, passará a ser compreendida como primariamente relacionada ao comportamento social?

\section{A PANDEMIA COVID-19 - Século XXI (2019- atual)}

A era pós-moderna, marcada pela globalização e importantes avanços na economia, trouxe com ela diversas facilitações, seja para o benefício da população, seja para a gênese e/ou desdobramentos de doenças que, no século XXI, se deslocam por meios diversos e facilmente se estabelecem em grandes aglomerados urbanos, enquanto via internet tornam-se especulação (Harper e Armelagos, 2010).

Nesse contexto, em dezembro de 2019 ocorre os primeiros casos do novo vírus Corona, o SARSCoV-2 (Severe Acute Respiratory Syndrome Coronavirus 2), em Wuhan, capital e maior cidade da província de Hubei, na China (Wang, 2020). O vírus, três meses depois, já em 206 países, haviam infectado mais de um milhão de pessoas, com mais de cinquenta mil mortes (WHO, 2020a) e, ainda agora, em franca expansão com graves perspectivas.
Essa nova Coronavirus Disease 2019 (COVID-19), com muitos aspectos ainda desconhecidos, apresenta taxa de letalidade em torno de 3 a 4\% (WHO,2020a), mais elevada do que os 2 a $3 \%$ (WHO, 2020b) da intimidadora Gripe Espanhola, e denota significativo número (5\%) de internações em unidades de terapia intensiva, mais alarmante se comparada a outras infecções respiratórias (Guan et al., 2020). Contrapondo a isso, $80,9 \%$ dos quadros da infecção em geral são considerados leves, com nenhum ou poucos sintomas (Zhi et al., 2020).

Devido à alta transmissibilidade, acelerado ritmo de propagação e poucas informações sobre o agente infeccioso, a Organização Mundial da Saúde (OMS) decretou em 31 de janeiro de 2020 estado de emergência para a saúde global (Dhama et al., 2020), uma crise tripla, assentada nos pilares comportamentais, sanitários e econômicos, num panorama que leva a COVID-19 a ser considerada a segunda maior crise do mundo moderno, atrás somente da Segunda Guerra Mundial (Netto, 2020).

É difícil, por enquanto, mensurar a quantidade de pessoas infectadas em todo o mundo, seja pelos $80 \%$ de pacientes assintomáticos (Ferretti et al., 2020), seja por não existir testes rápidos suficientes para avaliar toda a população. Todavia, por meio de sites, é possível em tempo quase real a monitorização da estimativa e do aumento dos casos em todo o globo, e estão em curso pesquisas de vacinas e medicamentos (Zhao et al., 2020). Na perspectiva de resultados não imediatos, o autocuidado e a responsabilidade são ainda mais importantes para que resultados efetivos ocorram (Carvalho, 2010).

Diante do avanço descontrolado do vírus, a população mundial reavalia suas práticas de higiene, em associação com a imposição da quarentena 
horizontal, fechamento de escolas, universidades e parte do comércio e serviços, contenção de casos suspeitos e resguardo dos grupos considerados de risco (Brooks et al., 2020; Ferguson et al., 2020). Isto tem propiciado uma súbita aproximação dos núcleos familiares (Bavel et al., 2020) e um expressivo aumento da utilização de redes sociais.

Esta pandemia se torna, então, diferente de todas as outras, devido à maciça presença das mídias sociais e pela infodemia, termo que caracteriza a excessiva quantidade e variedade de informações, até duvidosas e muitas vezes falsas, que dificultam orientações confiáveis representando riscos para a saúde global (Kalil e Santini, 2020).

Estudo realizado no final de 2019 pela União Internacional de Telecomunicações (UIT) estima que $53,6 \%$ da população mundial (equivalente a 4,1 bilhões de pessoas) está conectada de alguma forma à internet e, em geral, tende a adquirir informações tendenciosas, ignorar informações divergentes da maioria e formam grupos polarizados em torno das narrativas compartilhadas (Bessi et al., 2015; Zollo et al., 2017; Baronchelli, 2018; Vicario et al., 2016; Bail, 2018). Enquanto a presença de novas tecnologias de comunicação e informação facilitam e favorecem a vigilância e o manejo mais rápido e adequado de uma situação pandêmica, essa mesma disposição de tecnologias, facilita informações duvidosas e inadequadas, que repercutem seriamente no comportamento humano no contexto da pandemia.

Isto contribui e/ou desencadeia aumento de sintomas de estresse pós-traumático, confusão, raiva (Brooks et al., 2020; Cohen, 1995) e a veiculação de informações inadequadas contribui para o medo e insegurança da população que, mediante qualquer sintoma, desloca-se para unidades de saúde sobrecarregadas e acentua enormemente o risco de maior propagação da infecção.

De forma dinâmica, isso desafia as instituições da saúde mundial, visto que notícias falsas e informações imprecisas se espalham mais amplamente do que notícias baseadas em fatos e evidências (Vosoughi et al., 2018). No Vietnã e Coréia do Sul foi necessário a criação de leis que estabelecessem multas para quem espalhasse notícias falsas sobre a pandemia (Fleming, 2020; Mccurry, 2020), enquanto no Brasil foram disponibilizados aplicativos para dispositivos de telefonia móvel, como o Coronavírus-SUS, contendo informações das autoridades sanitárias (MS, 2020).

Portanto, ferramentas na Internet e mídias sociais criam novas perspectivas na vigilância de doenças, capturam dados em tempo real e contribuem para melhor resultado na saúde (Aiello et al., 2020), mas com pautas sócio comportamentais exacerbadas provocam reações emocionais, como medo e pânico que se instalam na população e facilitam pensamentos de soma zero, como acumular materiais de proteção além do necessário, enaltecendo o egoísmo e deixando de pensar nos outros (Bavel et al.,2020). E sendo comum populações culparem grupos não pertencentes à sua comunidade por alguma fatalidade, estar ameaçado por uma doença pode, frequentemente, desencadear reações etnocêntricas (Gurgel, 2013; Schaller e Neuberg, 2012), como as relatadas em países predominantemente brancos, onde houveram ataques a pessoas de etnia asiática (Russell, 2020).

Nessa conjuntura, é importante lembrar, tal como nos alertava John Stuart Mill (1991), que:

O povo que exerce o poder não é sempre o mesmo povo sobre quem o poder é exercido (...), a vontade do povo significa praticamente a vontade da mais numerosa e ativa parte do 
povo - a maioria, ou aqueles que logram êxito em se fazerem aceitar como a maioria. $\mathrm{O}$ povo, consequentemente, pode desejar oprimir uma parte de si mesmo, e precauções são tão necessárias contra isso quanto contra qualquer outro abuso de poder.

Outra variável notável é o fator cultural, responsável por diferenças nos números de infectados e óbitos, ou até mesmo na disseminação do SARSCoV-2 entre países distintos. Em culturas mais "restritivas", como Cingapura, Japão e China os indivíduos são mais comprometidos com obrigações e deveres, motivando o grupo a permanecer de acordo com as normas sociais impostas. Já em países como EUA, Itália e Brasil, de culturas consideradas "liberais", com normas sociais frágeis e permissivas, o egocentrismo, o desrespeito e descumprimento das orientações sanitárias são muito maiores (Kitayama et al., 2018; Betsch, 2017; Kraus e Kitayama, 2019; Gelfand et al., 2011; Gelfand et al., 2017).

No caso particular do Brasil, a franca polarização política, existente anteriormente ao início da pandemia, constituiu terreno fértil para a fragmentação e descoordenação das ações, acentuando um divisionismo no interior dos governos, e entre eles, tendo a sociedade papel de agente e vítima do falso conflito entre saúde e economia. Falso porque, numa pandemia exigindo forte isolamento social, essencial ao seu manejo, torna-se imperioso associar às medidas sanitárias arranjos e iniciativas econômicas, protetoras da população e dos negócios e serviços essenciais.

Então, considerar somente as posições governamentais como responsáveis pela dicotomia é incorreto, está apenas existe e sobrevive graças ao comportamento social, uma vez que:

A sociedade pode executar e executa os próprios mandatos; e, quando ela expede mandatos errados no lugar de mandatos certos, ou mandatos relacionados a coisas nas quais não se deve intrometer, pratica uma tirania social mais terrível do que muitas formas de opressão ou imposição políticas (Mill, 1991).

Assim, interfere na tomada de decisões e, consequentemente, nos desdobramentos da pandemia.

O quesito econômico é impactante, a interrupção abrupta do comércio global (Ayittey et al., 2020) causa desemprego e sobrecarga nos indicadores da fome. Segundo estudo realizado pela Organização Internacional do Trabalho (OIT), 81\% das 3,3 bilhões de pessoas que compõem a força de trabalho global, são afetadas pelo fechamento total ou parcial do local de trabalho, fazendo desaparecer $6,7 \%$ das horas de trabalho no $2^{\circ}$ trimestre de 2020 (ILO, 2020). O aumento da desigualdade social cria situações de maior exposição ao risco (Cockerham et al., 2017; Fothergill e Peek, 2004) como a utilização dos transportes coletivos aglomerados, moradores de rua sem condições básicas de higiene e sobrevivência, núcleos familiares sem saneamento básico em sua comunidade, dificultando dentre outras medidas lavar as mãos com frequência (Deitz e Meehan, 2019).

Embora existam atitudes solidárias, pois cenários pandêmicos tendem a destacar uma identidade comum aos indivíduos e um senso de destino compartilhado, por enfrentarem o mesmo risco (Bavel et al., 2020) isto não impede tentativas de priorizar e garantir a sobrevivência dos socialmente mais valorizados e economicamente mais abastados, ou os mais aptos e mais necessários para a economia, esquecendo-se ou negligenciandose dos cuidados com os mais vulneráveis (Santos, 2020).

Edgar Morin (2020) em recente entrevista ao jornal El País, afirma que esse mercado planetário não 
tem sabido suscitar, nem alimentar a fraternidade entre os povos, enquanto Santos (2020) adverte que:

Os três pilares de regulação das sociedades modernas são o Estado, o mercado e a comunidade, mas nos últimos quarenta anos, em detrimento do Estado e da comunidade, fora dada prioridade absoluta ao princípio do mercado, e o próprio Estado e a comunidade passaram a ser geridos e avaliados pela lógica do mercado.

Portanto, a pandemia mostra de maneira cruel como o projeto neoliberal, com aquiescência da sociedade, incapacitou o Estado para responder a emergências.

Este estudo - produzido em plena expansão dessa pandemia global, e com escassez de informações sobre o assunto, especialmente, sobre o vírus, sua dinâmica e impacto na população mundial, bem como na economia e na (re)organização do Estado e da sociedade -, reconhece que diversos pontos aqui abordados e discutidos podem se tornar obsoletos ou até equivocados, face à grande quantidade e velocidade de novos estudos e pesquisas em curso, no Brasil e mundo afora.

\section{CONCLUSÃO}

Atravessar períodos difíceis, como nas pandemias descritas, e resgatar suas histórias, contextos sociais e as reações da população pode ser uma fonte de inesgotáveis aprendizados para o enfrentamento de crises, como também para gerar lições ativas sobre o futuro.

Com uma visão multicausal para o estudo das doenças, especificamente nestas três pandemias abordadas, é evidente a relação dos meios de transporte e de comunicação, na maior e mais rápida propagação dos microrganismos, tanto entre os países, quanto dentro deles.
A globalização, mesmo favorecendo a cooperação e troca de informações e produção de pesquisas, de vacinas, de medicamentos e de conhecimento do modus operandi do microrganismo, exerce um papel crucial na disseminação de doenças, face à dinâmica movimentação/locomoção de pessoas ou de produtos e, especialmente, à resultante precarização de condições de vida e trabalho.

Então, se os conhecimentos científicos e a disponibilidade de recursos são essenciais no enfrentamento e superação das pandemias, o conhecimento da influência do comportamento humano/social é determinante para abreviar sua solução e, principalmente, para consolidar lições aprendidas, sendo uma delas o entendimento do papel importante de políticas de Estado efetivas, como medidas de desenvolvimento e protetoras da população.

Portanto, na prevenção quanto no enfrentamento de pandemias, ressalta-se a importância e necessidade do emprego de outras abordagens e compreensões, especialmente no campo das ciências sociais, para melhor reconhecer e administrar a influência do comportamento humano e social.

Todos os autores declararam não haver qualquer potencial conflito de interesses referente a este artigo.

\section{REFERÊNCIAS}

AIELLO, A.E.; RENSON, A.; Zivich, P.N. Social Media- and Internet-Based Disease Surveillance for Public Health. Annu. Rev. Public Health. v.41, p.101-118, 2020.

AYITTEY, F.K.; AYITTEY, M.K.; CHIWERO, N.B.; KAMASAH, J.S.; DZUVOR, C. Economic Impacts of Wuhan 2019-nCoV on China and the World. J Med Virol.v. 92, n.5, p.473-475, 2020. 
BAIL， C.A.; ARGYLE, L.P.; BROWN, T.W.; BUMPUS, J.P.; CHEN H.; HUNZAKER, M.B.F.; LEE, J.; MANN, M.; MERHOUT, F.; VOLFOVSKY, A. Exposure to opposing views on social media can increase political polarization. Proc Natl Acad Sci U S A. v.115, n.37, p.9221, 2018.

BARONCHELLI, A. The emergence of consensus: a primer. R Soc Open Sci. v.5, n.2, p.172189, 2018.

BARRIOS, J.M.; HOCHBERG, Y.V. Risk Perception Through the Lens of Politics in the Time of the COVID-19 Pandemic. NBER Work Pap Ser. n. 27008, 2020.

BARRY, J.M. The Great Influenza: The Story of the Deadliest Pandemic in History. London: Penguin Books Ltd; 2004.

BAUCH, C.T.; GALVANI, A.P. Epidemiology. Social factors in epidemiology. Science. v.342, n.6154, p.47-49, 2013.

BAVEL, J.J.V.; BAICKER, K.; BOGGIO, P.S.; CAPRARO, V.; CICHOCKA, A.; CIKARA, M.; CROCKETT, M.J.; CRUM, A.J.; DOUGLAS, K.M.; DRUCKMAN, J.N.; DRURY, J.; DUBE, O.; ELLEMERS, N.; FINKEL, E.J.; FOWLER, J.H.; GELFAND, M.; HAN, S.; HASLAM, A.S.; JETTEN, J.; KITAYAMA, S.; MOBBS, D.; NAPPER, L.E.; PACKER, D.J.; PENNYCOOK, G.; PETERS, E.; PETTY, R.E.; RAND, D.G.; REICHER, S.D.; SCHANALL, S.; SHARIFF, A.; SKITKA, L.J.; SMITH, S.S.; SUNSTEIN, C.R.; TABRI， N.; TUCKER， J.; LINDEN， S.V.D.; LANGE, O.V.; WEEDEN, K.A.; WOHL, M.J.A.; ZAKI, J.; ZION, S.R.; WILLER, R. Using social and behavioural science to support COVID-19 pandemic response. Nat Hum Behav, 2020.

BERTUCCI, L.M. A onipresença do medo na influenza de 1918. Varia hist. v. 25, n.42, p. 457-75, 2009.

BERTUCCI, L.M. Influenza, medicina enferma. Ciência e práticas de cura na época da gripe espanhola em São Paulo. Campinas-SP: Editora da Unicamp; 2004.

BESSI, A.; COLETTO, M.; ALEXANDRU, G.; DAVIDESCU, A.; SCALA, A.; CALDARELLI, G.; QUATTROCIOCCHI, W. Science vs conspiracy: Collective narratives in the age of misinformation.

PloS One. v.10, n.2, p.0118093, 2015.
BETSCH, C. On the benefits of explaining herd immunity in vaccine advocacy. Nat. Hum. Behav. v.1, p.1-6, 2017.

BROOKS, S.K.; WEBSTER, R.K.; SMITH, L.E. The psychological impact of quarantine and how to reduce it: rapid review of the evidence. Lancet. v.395, n.10227, p. 912-920, 2020.

CARVALHO, M. 2010. Ciclo de conferências do ISCS-N: pandemias ou pânico. Disponível em: http://www.cienceduc.com/iscs-n/eventos/vi-cicloconferencias.html. Acesso em: 20/04/2020.

CHALTON, N.; MACARFLE, M. A história do século 20 para quem tem pressa. $1^{\circ} \mathrm{ed}$. Rio de Janeiro: Valentina; 2017.

COCKERHAM, W.C.; HAMBY, B.W.; OATES, G.R. The Social Determinants of Chronic Disease. Am. J. Prev. Med. v.52, p.S5-S12, 2017.

COHEN, S. Psychological Stress and Susceptibility to Upper Respiratory Infections. Am J Respir Crit Care Med. v.152, n.4, p.S53-S58, pt.2, 1995.

CONTI, T.V. 2020. Crise Tripla do Covid-19: um olhar econômico sobre políticas públicas de combate à pandemia. Disponível em: http://bit.ly/covid19crisetriplaCopy . Acessado em: 30/04/2020.

CROSBY, A. The America's forgotten pandemic: The influenza of 1918. $2^{\mathrm{a}}$ ed. Cambridge: Cambridge University Press; 1999.

DEITZ, S.; MEEHAN, K. Plumbing Poverty: Mapping Hot Spots of Racial and Geographic Inequality in U.S. Household Water Insecurity. Ann. Am. Assoc. Geogr. v.109, p.1092-1109,2019.

DELACY, M. The conceptualization of influenza in eighteenth-century Britain: specificity and contagion. Bull Hist Med Suppl. v.67, n.1, p.75-117, 1993.

DHAMA, K.; SHARUN, K.; TIWARI, R.; SCHUBHANKAR, S.; BHAT, S.; MALIK, Y.S.; SINGH, K.P.; CHAICUMPA, W.; BONILLAALDANA, D.K.; RODRIGUEZ-MOLALES, A.J. Coronavirus disease 2019-COVID-19. Preprints. v.2020030001, 2020.

EPSTEIN, J.M. Modelling to contain pandemics. Nature. v.460, n.7256, p.687, 2009. 
FERGUSON, N. Capturing human behaviour. Nature. v.446, n.7137, p.733-733, 2007.

FERGUSON, N.M.; LAYDON, D.; NEDJATIGILANI, G.; IMAI, N.; AINSLIE, K.; BAGUELIN, M.; BHATIA, S.; BOONYASIRI. A.; CUCUNUBÁ, Z.; CUOMO-DANNENBURG, G.; DIGHE, A.; DORIGATTI, I.; FU, H.; GAYTHORPE, K.; GREEN, W.; HAMLET, A.; HINSLEY, W.; OKELL, L.C.; ELSLAND, S.V.; THOMPSON, H.; VERITY, R.; VOLZ, E.; WANG, H.; WANG, Y.; WALKER, P.G.T.; WALTERS. C.; WINSKILL, P.; WHITTAKER, C.; DONNELLY, C.A.; RILEY, S.; GHANI, A.C. Report 9: Impact of nonpharmaceutical interventions (NPIs) to reduce COVID-19 mortality and healthcare demand. Bull Math Biol. v.82, n.4, p.52, 2020.

FERRETTI, L.; WYMANT, C.; KENDALL, M.; ZHAO, L.; NURTAY, A.; ABELER-DORNER, L.; PARCKER, M.; BONSALL, D.; FRASER, C. Quantifying SARS-CoV-2 transmission suggests epidemic control with digital contact tracing. Science. v.368, n.6491, 2020.

FLECKNOE, D.; WAKEFIELD, B.C.; SIMMONS, A. Plagues \& wars: the 'Spanish Flu' pandemic as a lesson from history. Med Confl Surviv. v.34, n. 2, p.61-68, 2018.

FLEMING, S. 2020. Viet Nam shows how you can contain COVID-19 with limited resources. World Economic Forum. Disponível em: https://www.weforum.org/agenda/2020/03/vietnamcontain-covid-19-limited-resources/ Acessado em: $27 / 04 / 2020$

FOTHERGILL, A.; PEEK, L.A. Poverty and Disasters in the United States: A Review of Recent Sociological Findings. Nat. Hazards. v.32, p.89110, 2004.

FUNK, S.; SALATHÉ, M.; JANSEN, V.A.A. Modelling the influence of human behaviour on the spread of infectious diseases: a review. J. R. Soc. Interface. v.7:, p.1247-1256, 2010.

GELFAND, M.J.; HARRINGTON, J.R.; JACKSON, J.C. The Strength of Social Norms Across Human Groups. Perspect. Psychol. Sci. v.12, p.800-809, 2017.

GELFAND, M.J.; RAVER, J.L.; NISHII, L.; LESLIE, L.M.; LUN, J.; LIM, B.C.; DUAN, L.; ALMALIACH, A.; ANG, S.; ARNADOTTIR, J.;
AYCAN, Z.; BOEHNKE, K.; BOSKI, P.; CABECINHAS, R.; CHAN, D.; CHHOKAR, J.; D’AMATO, A.; FERRER, M.; FISCHLMAYR, I.C.; FISCHER, R.; FULOP, M.; GEORGAS, J.; KASHIMA, E.S.; KASHIMA, Y.; KIM, K.; LEMPEREUR, A.; MARQUEZ, P.; OTHMAN, R.; OVERLAET, B.; PANAGIOTOPOULOU, P.; PELTZER, K.; PEREZ-FLORIZNO, L.R.; PONOMARENKO, L.; REALO, A.; SCHEI, V.; SCHMITT, M.; SMITH, P.B.; SOOMRO, N.; SZABO, E.; TAVEESIN, N.; TOYAMA, M.; DE VLIERT, E.V.; VOHRA, N.; WARD, N.; WARD, C.; YAMAGUCHI, S. Differences Between Tight and Loose Cultures: A 33-Nation Study. Science. v.32, p.1100-1104, 2011.

GONZALEZ, R.L. Curar, persuadir y gobernar: la construcción histórica de la profesión médica en Buenos Aires, 1852-1886. Madrid: CSIC; 1999.

GONZATTO, M.; CORSO, M.; SENHORAS, E.M.; SEGATA, J.; NETO, A.M.; VERONESE, M.V. 2020. Mudanças de comportamento, na economia e no trabalho: como as pandemias transformam o mundo. GaúchaZH. Disponível em: https://works.bepress.com/eloi/581/. Acessado em: $20 / 03 / 2020$

GREEN, B.N.; JOHNSON, C.D.; ADAMS, A. Writing narrative literature reviews for peerreviewed \njournals: secrets of the trade. J Chiropr Med. v.5, n.3, p.101-117, 2006.

GUAN, W-J.; NI, Z-Y.; HU, Y.; LIANG, W-H.; OU, C-Q.; HE, J-X.; LIU, L.; SHAN, H.; LEI, C-L.; HUI, D.S.C.; DU, B.; LI, L-J. Clinical Characteristics of Coronavirus Disease 2019 in China. N Engl J Med. v.382, p.1708-1720, 2020.

GURGEL, C.B.F.M. 1918: a gripe espanhola desvendada? Rev Soc Bras Clin Med. v.11, n.4, p.380-5, 2013.

HARPER, K.; ARMELAGOS, G. The changing disease-scape in the third epidemiological transition. Int J Environ Res Public Health. v.7, n.2, p.675697, 2010.

HERZLICH, C. Saúde e doença no início do século XXI: entre a experiência privada e a esfera pública. Physis. v.14, n.2, p.383-394, 2004.

HOLMBERG M. The ghost of pandemics past: revisiting two centuries of influenza in Sweden. Med Humanit . v.43, n.3, p.141-147, 2017. 
HOWARD-JONES, N. Cholera nomenclature and nosology: A historical note. Bull World Health Organ. v.51, p.317-324, 1979.

INTERNATIONAL LABOUR ORGANIZATION (ILO) .2020. COVID-19 and the world of work. Second edition. Updated estimates and analysis. Disponível em: https://www.ilo.org/wcmsp5/groups/public/@ dgrepo rts/@dcomm/documents/briefingnote/wcms_740877 .pdf Acessado em: 28/04/2020

JONES, D.S. History in a Crisis - Lessons for Covid19. N Engl J Med. v.382, p.1681-1683, 2020

KALIL, I.; SANTINI, R.M. 2020. "Coronavírus, Pandemia, Infodemia e Política". Relatório de pesquisa. Divulgado em 01 de abril de 2020. 21p. São Paulo / Rio de Janeiro: FESPSP/UFRJ. Disponível em:

https://www.fespsp.org.br/store/file_source/FESPSP /Documentos/Coronavirus-e-infodemia.pdf

Acessado em: 28/04/2020

KITAYAMA, S.; PARK, J.; MIYAMOTO, Y.; DATE, H.; BOYLAN, J.M.; MARKUS, H.R.; KARASAWA, M.; KAWAKAMI N.; COE, C.L.; LOVE, G.D.; RYFF, C.D. Behavioral Adjustment Moderates the Link Between Neuroticism and Biological Health Risk: A U.S.-Japan Comparison Study. Pers. Soc. Psychol. Bull . v.44, p.809-822, 2018.

KOLATA G. Flu: The History of the great influenza pandemic of 1918 and the search for the virus that caused it. London: Macmillan; 2000.

KRAUS, B.; KITAYAMA, S. Interdependent selfconstrual predicts emotion suppression in Asian Americans: Na electro-cortical investigation. Biol. Psychol. v.146, p.107733, 2019.

LODGE, E.K.; SHATZ, A.M.; DRAKE, J.M. Protective Population Behavior Change in Outbreaks of Emerging Infectious Disease. bioRxivorg. v.921536, 2020.

MACPHERSON J. Annals of Cholera from the Earliest Periods to the Year 1817. $2^{\circ}$ ed. London: HK Lewis; 1884.

MATTOS, M.F.D. O sentido da Modernidade no imaginário do século XIX. Associação Brasileira de Estudos de Pesquisas em Moda. v.3, n.6, p.96-103.
MCCURRY, J. 2020. South Korea cracks down on fake news about spread of coro-navirus. The Guardian. Disponível em: https://www.theguardian.com/world/2020/jan/30/sou th-korea-cracks-down-on-fake-news-about-spreadof-coronavirus Acessado em: 29/04/2020.

MILL J.S. Sobre a liberdade. Tradução e prefácio de Alberto da Rocha Barros; Apresentação de Celso Lafer. 2a ed. Petrópolis, RJ: Vozes, p.28;1991.

MINISTÉRIO DA SAÚDE (MS). 2020. Aplicativo Coronavírus SUS agora envia mensagens de alertas aos usuários. Disponível em: https://www.saude.gov.br/noticias/agenciasaude/46628-aplicativo-coronavirus-sus-agoraenvia-mensagens-de-alertas-aos-usuarios Acessado em: $20 / 04 / 2020$

MONTO, A.S.; WEBSTER, R.G. Influenza pandemics: history and lessons learned. $2^{\circ} \mathrm{ed}$. Oxford: Wiley Blackwel; p.20-34, 2013

MORIN, E. 2020. LA CRISIS DEL CORONAVIRUS Disponível em: https://elpais.com/cultura/2020-04-11/edgar-morinvivimos-en-un-mercado-planetario-que-no-hasabido-suscitar-fraternidad-entre-los-pueblos.html. Acessado em: 27/04/2020.

NETTO, F.F. A natureza humana e suas formas de exteriorização em tempos de pandemia da COVID19. Boletim de Conjuntura. v.2, n.4, 2020.

NICKOL, M.E.; KINDRACHUK, J. A year of terror and a century of reflection: perspectives on the great influenza pandemic of 1918-1919. BMC Infect Dis. v.19, n.1, p.117, 2019.

OMRAN, A.R. The epidemiologic transition: a theory of the epidemiology of population change. Milbank Q. v.83, n.4, p.731-757, 2005.

PACINI, F. Microscopic observations and pathological deductions on Asiatic cholera [in Italian]. Gazz Med Ital . v.4, n.50, p. 397-401 e v. 4, n.51, p.405-412, 1854.

PASCUAL, C.M. The cholera epidemic as condenser of meanings: urban cultures, clinical narratives, and hygiene policies in Rosario, Argentina, 1886-1887. Hist Cienc Saude Manguinhos. v.24, n.2, p.295311, 2017. 
POLLITZER, R. History of the disease. In R. Pollitzer (ed.), Cholera. Geneva:World Health Organization. No. 43, p. 11-50, 1959.

REZENDE, J.M.; MORAES, V.A.D.; PERINI, G.E. Seara de Asclépio: uma visão diacrônica da medicina [Recurso eletrônico]. $2^{\circ}$ ed. Goiânia: Editora UFG; 2018.

RODRÍGUEZ, B. El cólera. Buenos Aires: Buffet and Bosch; 1889.

RUSSELL, A. The Rise of Coronavirus Hate Crimes. The New Yorker.

SANTOS, B.S. A cruel pedagogia do vírus. Boitempo; 2020.

SANTOS, L.A.C. Um século de cólera: itinerário do medo. Physis . v.4, n.1, p.79-110, 1994.

SCHALLER, M.; NEUBERG, S.L. Danger, Disease, and the Nature of Prejudice(s). Adv Exp Soc Psychol. v.46,p.1-54, 2012.

SCHRECKER, T. The Ashgate research companion to the globalization of health. Routledge; 2012.

SEQUEIRA, A. A pneumónica. Arch Med Intern. v.8, n.1, p.49-55, 2001.

SHORT, K.R.; KEDZIERSKA, K.; VAN DE SANDT, C.E. Back to the future: lessons .learned from the 1918 influenza pandemic. Front Cell Infect Microbiol. v.8, p.343, 2018.

SILVEIRA, A.J.T. A medicina e a influenza espanhola de 1918. Tempo. v.10, n.19, p.91-105, 2005.

TAUBENBERGER, J.K.; MORENS, D.M. 1918 Influenza: the Mother of All Pandemics. Emerging Infectious Diseases. Rev Biomed. v.7, n.1, p.69-79, 2006.

TOGNOTTI, E. "Scientific triumphalism and learning from the facts: bacteriology and the spanish flu challenge of 1918". Soc Hist Med. v.16, n.1, p.97110, 2003.

VICARIO, M.D.; VIVALDO, G.; BESSI, A.; ZOLLO, F.; SCALA, A.; CALDARELLI, G.; QUATTROCIOCCHI, W. Echo chambers: Emotional contagion and group polarization on facebook. Sci Rep. v.6, p.37825, 2016.
VICENTINI, C.B.; ALTIERI, L.; MANFREDINI, S. The treatment of cholera in Ferrara (Italy): the European epidemic scenery in the first half of the 19th century. Pharm Hist. v. 41, n.3, p.34-41, 2011.

VOSGERAU, D.S.R.; ROMANOWSKI, J.P. Estudos de revisão: implicações conceituais e metodológicas. Rev Dialogo Educ. v.14, n.474, p.165, 2014.

VOSOUGHI, S.; ROY, D.; ARAL, S. The spread of true and false news online. Science. v. 359, n.6380, p.1146-1151, 2018.

WANG, C.; HORBY, P.W.; HAYDEN, F.G.; GAO, G.F. A novel coronavírus outbreak of global health concern. Lancet. v.395, n.10223, p. 470-473, 2020.

WHITE, P. Epidemics and Pandemics: Their Impacts on Human History. Reference Reviews; 2006.

WOKAUNN, M.; FATOVIĆ-FERENCIĆ, S.; JURIĆ, I.; BEKIĆ, M. God's Punishment or Bad Strategy: Anti-Epidemic Measures in the Lower Neretva Basin at the Time of Cholera in 1886. Coll. Antropol. v.36, n.3, p.987-995, 2012.

World Health Organization (WHO). 2020. DirectorGeneral's opening remarks at the media briefing on COVID-19 - 3 March 2020. Disponível em: https:// www.who.int/dg/speeches/detail/whodirectorgeneral-s-opening-remarks-at-the-mediabriefing-oncovid-19---3-march-2020 Acessado em: 16/04/2020.

World Health Organization (WHO). 2019. Pandemic Influenza preparedness and response: a WHO guidance document. Disponível em: https://apps.who.int/iris/bitstream/ handle/10665/44123/9789241547680_eng.pdf Acessado em : 16/04/2020.

ZHAO, S.; LIN, Q.; RAN, J.; MUSA, S.S.; YANG, G.; WANG, W.; LOU, Y.; GAO, D.; HE, D.; WANG, M.H. Preliminary estimation of the basic reproduction number of novel coronavirus (2019nCoV) in China, from 2019 to 2020: A data-driven analysis in the early phase of the outbreak. Int $\mathbf{J}$ Infect Dis. v.92, p.214-217, 2020.

ZHI, Z.L.X.B.X.Z. Epidemiology Working Group for NCIP Epidemic Response, Chinese Center for 
Disease Control and Prevention. v.41, n.2, p.145-151, 2020.

ZOLLO, F.; BESSI, A.; VICARIO, M.D.; SCALA, A.; CALDARELLI, G.; SHEKHTMAN, L.; HAVLIN, S.; QUATTROCIOCCHI, W. Debunking in world of tribes. PloS One. v.12, n.7, 2017. 